\title{
BMJ Open Association of outpatient ACE inhibitors and angiotensin receptor blockers and outcomes of acute respiratory illness: a retrospective cohort study
}

\author{
Molly Moore Jeffery (D) , , Nathan W Cummins, ${ }^{3}$ Timothy M Dempsey, ${ }^{4}$ \\ Andrew H. Limper, ${ }^{5,6}$ Nilay D Shah, ${ }^{1,6}$ Fernanda Bellolio ${ }^{2}$
}

To cite: Jeffery MM, Cummins NW, Dempsey TM, et al. Association of outpatient ACE inhibitors and angiotensin receptor blockers and outcomes of acute respiratory illness: a retrospective cohort study. BMJ Open 2021;11:e044010. doi:10.1136/ bmjopen-2020-044010

- Prepublication history and additional material for this paper are available online. To view these files, please visit the journal online (http://dx.doi. org/10.1136/bmjopen-2020044010).

Received 24 August 2020 Revised 09 February 2021 Accepted 01 March 2021

Check for updates

(C) Author(s) (or their employer(s)) 2021. Re-use permitted under CC BY-NC. No commercial re-use. See rights and permissions. Published by BMJ.

For numbered affiliations see end of article.

Correspondence to Dr Molly Moore Jeffery; jeffery.molly@mayo.edu

\section{ABSTRACT}

Objectives Evaluate associations between ACE inhibitors (ACEis) and angiotensin receptor blockers (ARBs) and clinical outcomes in acute viral respiratory illness (AVRI). Design Retrospective cohort analysis of claims data. Setting The USA; 2018-2019 influenza season. Participants Main cohort: people with hypertension (HTN) taking an ACEi, ARB or other HTN medications, and experiencing AVRI. Falsification cohort: parallel cohort receiving elective knee or hip replacement.

Main outcome measures Main cohort: hospital admission, intensive care unit, acute respiratory distress (ARD), ARD syndrome and all-cause mortality. Falsification cohort: complications after surgery and all-cause mortality.

Results The main cohort included 236843 episodes of AVRI contributed by 202629 unique individuals. Most episodes were in women (58.9\%), 81.4\% in people with Medicare Advantage and $40.3 \%$ in people aged $75+$ years. Odds of mortality were lower in the ACEi (0.78 (0.74 to 0.83$)$ ) and ARB (0.64 (0.61 to 0.68$)$ ) cohorts compared with other HTN medications. On all other outcomes, people taking ARBs (but not ACEis) had a $>10 \%$ reduction in odds of inpatient stays compared with other HTN medications.

In the falsification analysis $(\mathrm{N}=103353)$, both ACEis (0.89 (0.80 to 0.98)) and ARBs (0.82 (0.74 to 0.91)) were associated with decreased odds of complications compared with other HTN medications; ARBs (0.64 (0.47 to 0.87$)$ ) but not ACEis ( 0.79 (0.60 to 1.05$)$ ) were associated with lower odds of death compared with other HTN medications.

Conclusions Outpatient use of ARBs was associated with better outcomes with AVRI compared with other medications for HTN. ACEis were associated with reduced risk of death, but with minimal or no reduction in risk of other complications. A falsification analysis conducted to provide context on the possible causal implications of these findings did not provide a clear answer. Further analysis using observational data will benefit from additional approaches to assess causal relationships between these drugs and outcomes in AVRI.
Strengths and limitations of this study

- This study draws on a large and diverse cohort of US residents including people with commercial insurance and Medicare Advantage.

- It builds on prior literature by rigorously comparing baseline characteristics of people taking different types of hypertension medications and by using a falsification test.

- We are unable to directly assess blood pressure control, which could affect mortality.

- The cohort does not include uninsured people or people with Medicaid or other insurance types.

- We are unable to make causal claims about the relationship between ACE inhibitors or angiotensin receptor blockers and outcomes with acute viral respiratory illness.

\section{INTRODUCTION}

Theoretical concerns have been raised about the possibility that ACE inhibitors (ACEis) and angiotensin receptor blockers (ARBs) may increase susceptibility to SARS-CoV-2. ${ }^{1}$ Currently, no high-quality clinical evidence suggests that patients with COVID-19 have worse outcomes while continuing to take ACEis or ARBs. Furthermore, ACEis and ARBs have been proposed to have protective effects based on their mechanism of action, ${ }^{1} 2$ and current recommendations by major clinical societies are that patients should continue treatment with these agents if there is no other reason for discontinuation. ${ }^{3-7}$ Clinical trials are underway to assess safety of continued use of ARBs and ACEis in people with COVID-19 and efficacy of these drug treatments for COVID-19. ${ }^{8}$

ACEis block the activity of ACE, which is part of the renin-angiotensin system that converts angiotensin I to angiotensin II. 
ARBs inhibit the angiotensin II type 1 receptor. Both classes of medications decrease blood pressure through inhibition of the renin-angiotensin-aldosterone system (RAAS) which plays a crucial role in the pathophysiology of hypertension (HTN) through its vasoconstrictive, proinflammatory and pro-oxidative effects. ${ }^{1}$ This system is also implicated in infection and disease associated with both SARS-CoV-1 and SARS-CoV-2. ${ }^{9}$ ACE2 is expressed in cells from the heart, kidneys and lungs and is the principal target cells for SARS-CoV-2. ACE2 in the alveolar epithelial cells of the lungs has been associated with respiratory distress secondary to increased permeability in the lungs seen in COVID-19 disease. ${ }^{9}$

Dysregulation of the RAAS may play a key role in the pathophysiology of acute respiratory distress syndrome (ARDS) both in association with COVID-19 and other aetiologies ${ }^{1}$ as severe ARDS secondary to impaired ACE2 activity has been identified in other viral pneumonias. ${ }^{10}$ Previous reports attributed a critical role in regulating HTN and acute lung injury caused by viruses, such as SARS $^{11}$ and influenza $(\mathrm{H} 7 \mathrm{~N} 9)^{12}$ to the RAAS. Following this physiology, the use of RAAS inhibitors like ACEis and ARBs will affect ACE2 expression. ${ }^{9}$ The advent of COVID-19 gives additional urgency to the question of whether ACEis or ARBs are protective in lower respiratory infections. A living systematic review currently reports that ACEi and ARB use is not associated with more severe COVID-19 disease, ${ }^{8}$ though the published studies are all observational, many with small sample sizes and drawing from a single healthcare system.

The objectives of this study were to evaluate associations between patients taking ACEis and ARBs and their clinical outcomes after an acute viral respiratory illness (AVRI), with attention to causal inference using a falsification test to attempt to distinguish the plausibility of a causal effect. We compare severity of illness and mortality in AVRI across cohorts of patients with HTN using ACEis, ARBs and other HTN medications. We then repeat the exercise in a cohort hypothesised to have no mechanism for better outcomes with ACEis or ARBs: people receiving elective hip or knee replacement surgeries.

\section{METHODS}

We adhered to the REporting of studies Conducted using Observational Routinely collected health Data statement. $^{13}$

\section{Study design and setting}

We conducted an analysis of de-identified administrative claims data from the OptumLabs Data Warehouse (OLDW), focusing on episodes of AVRI during the 20182019 influenza season, defined by the Centers for Disease Control and Prevention as spanning 30 September 2018 through 18 May 2019. ${ }^{14}$ The OLDW includes medical and pharmacy claims, laboratory results, and enrolment records for commercial and Medicare Advantage (MA) enrollees. ${ }^{15}$ The database contains longitudinal health information on enrollees and patients, representing a diverse mixture of ages, ethnicities and geographical regions across the USA.

\section{Patient involvement}

No patients were involved in setting the research question or the outcome measures, nor were they involved in developing plans for design or implementation of the study. No patients were asked to advise on interpretation or writing up of results. The results will be disseminated to the relevant patient community through press releases, media engagement, and social media posts explaining the study and its findings.

\section{Cohort}

We created a cohort of patients with one or more episodes of AVRI with an initial date of service between 30 September 2018 and 18 May 2019. Episodes started on the first date on which a patient received healthcare services coded with one of the AVRI diagnosis codes (the index date) and ended the last day before patients began a 30-day period with no encounters coded with an AVRI diagnosis. Codes for AVRI were identified using a series of diagnosis codes that represent viral causes of respiratory illness, including bronchitis, pneumonia, influenza, influenza-like illness and lower respiratory infections; bacterial infection diagnosis codes were excluded. The complete list of AVRI codes is available in online supplemental table 1 .

We limited the cohort to people with continuous enrolment in both medical and pharmacy insurance benefits for at least 180 days before the beginning of the episode; this period of prior insurance enrolment was used to ascertain patient comorbidities. Because some HTN medications are also used for other conditions, we limited the cohort to people who met claims criteria for complicated or uncomplicated HTN, as described below.

The cohort of interest is people taking an ACEi or an ARB. People taking other HTN medications comprise the comparison cohort. Tables include descriptive measures for both the HTN medication cohorts and for people with AVRI who are not taking any HTN medications; descriptions of this latter group are provided only for context: regression analyses exclude people not taking HTN medications.

\section{Variables}

We used insurance enrolment information to determine patient age, gender, state of residence and insurance type (commercial or MA).

\section{HTN and comorbidities}

We determined patient comorbidities using diagnoses from claims for healthcare services received in the period from 180 days before the index date up to and including the index date. HTN was classified hierarchically as uncomplicated or complicated (ie, a person with codes for both uncomplicated and complicated HTN was classified as having complicated HTN). Other chronic diseases 
that could affect patient outcomes with AVRI were defined using previously published lists of diagnosis codes for chronic obstructive pulmonary disease, diabetes (hierarchical: no diabetes, diabetes without complications, diabetes with complications), asthma, cancer, coronary artery disease, chronic kidney disease and chronic liver disease. ${ }^{16}{ }^{17}$ We required one inpatient or two outpatient dates of service with a diagnosis from the comorbidity list to assign a comorbidity to a patient.

\section{HTN medications}

We developed a comprehensive list of medications used to treat HTN from a clinical reference guide to HTN treatment, ${ }^{18}$ then identified those drugs in a comprehensive table of National Drug Code codes used during the study period. Pharmacy fill claims for these drugs were retrieved for the 90 days leading up to the index date and used to determine whether the patient was using an ACEi, ARB, and/or other HTN medications, or no HTN medication. People taking both an ACEi (or ARB) and some other classes of medication (beta-blocker, diuretic and so on) were classified as ACEi (or ARB) users. People not using an ACEi or ARB were classified as other HTN medication users. A small number of people had fills for both an ACEi and an ARB during the 90 days before their index dates. We excluded this group from further analysis.

\section{Outcomes}

We specified five outcomes that represented more serious cases of AVRI: hospital admission for one or more nights, any time spent in the intensive care unit (ICU) or coronary care unit (CCU) during an admission (defined as services with an ICU or CCU revenue code on the inpatient claim), any diagnosis of acute respiratory distress while in the hospital (ICD-10 code: R06.03), any diagnosis of ARDS while in the hospital (ICD-10 code: J80) and mortality. To protect patient privacy, only the month and year of death are available in the OLDW. We captured deaths in the same or following calendar month in the mortality outcome. For example, if an episode of AVRI ended on 5 January 2019, death in January 2019 or February 2019 was included in the mortality measure. Mortality was identified using a combination of data from the Death Master File from the Social Security Administration, from claims (ie, discharge status was 'deceased'), and from insurance enrolment records. A small number of people had inconsistent death information $(\mathrm{N}=410(0.17 \%)$ in the AVRI cohort and $\mathrm{N}=170(0.16 \%)$ in the falsification cohort) indicating death more than 1 calendar month before surgery. These people were excluded from the mortality analysis but included in other outcome analyses.

\section{Analysis}

We used logistic regression to calculate adjusted ORs for each outcome across the medication groups (ACEi, ARB, other HTN medications) while adjusting for observed demographic and clinical characteristics (female sex, age (categorical), insurance type (Medicare Advantage vs commercial), census division, race/ethnicity, binary variables for comorbidities (coronary artery disease, congestive heart failure, chronic pulmonary disease, renal failure, liver failure, metastatic cancer, lymphoma and non-metastatic solid tumour), and hierarchical comorbidity variables for HTN (uncomplicated HTN, complicated HTN) and diabetes mellitus (DM) (no DM, DM without complications, DM with complications). All regressions were specified with Huber/White SEs, clustered on patient ID, to adjust SEs for correlation due to multiple illness episodes for some patients. Complete regression results in supplemental materials include episode count, number of unique patients, model pseudo- $\mathrm{R}^{2}$ and the number of outcome events observed in the analysis cohort.

To assess the similarity of the medication cohorts on demographic and clinical characteristics, we calculated unadjusted risk ratios (RRs) comparing the ACEi cohort with the ARB cohort and comparing the combined ACEi or ARB cohort with the other HTN medications cohort. These RRs were calculated for each binary or categorical variable value. For example, for the binary variable female versus not female sex, we calculated the proportion of the ACEi cohort members who were female $\left(\mathrm{ACEi}_{\mathrm{f}}\right)$ and the proportion of the ARB cohort members who were female $\left(\mathrm{ARB}_{\mathrm{f}}\right)$, then calculated the RR $\mathrm{ACEi}_{\mathrm{f}} / \mathrm{ARB}_{\mathrm{f}}$ along with its $\mathrm{CI}$ and the $\mathrm{p}$ value for the hypothesis test that the $\mathrm{RR}=1$. For categorical variables, separate RRs were calculated for each value (eg, proportion age $85+$ years)

The descriptive analysis encompasses a large number of comparisons. To limit the overall type I error across these comparisons, we used the Holm method to estimate $\mathrm{p}$ values corrected for multiple comparisons, with a familywise error rate of $0.05 .^{19}{ }^{20}$ In the analysis of differences in cohort demographic and clinical characteristics, the family of comparisons is defined as the complete set of comparisons by cohort (ACEi vs ARB on all characteristics and ACEi or ARB vs other on all characteristics): a total of 72 comparisons in the AVRI cohort and a total of 70 comparisons in the falsification cohort.

\section{Falsification analysis}

To better understand the potential causal implications of our results, we decided a priori to conduct a falsification analysis. A falsification hypothesis is a claim, distinct from the one being tested, which is not causally related to the intervention in question. Its testing uses more similar statistical methods than the primary analysis. ${ }^{21}$ Falsification analysis helps to support the validity of observational studies. The idea behind a falsification test in this context is to select a condition that would not be expected to be causally affected by the treatment being studied, then assess whether this alternative condition shows a treatment effect. If no treatment effect is seen with the alternative condition, it supports, but does not prove, that there may be a causal treatment effect for the primary treatment studied. If the falsification analysis does show a 
treatment effect in the alternative condition, it may indicate that the treatment effect for the main analysis is a result of unmeasured confounding; a failed falsification analysis may also indicate there is a treatment effect in the falsification cohort. A successful falsification analysis can strengthen the causal claims underlying observational studies by demonstrating that the treatment effect is at least partially specific to the condition being studied rather than solely a reflection of unmeasured differences in underlying health.

The cohort selected for the falsification analysis was elective knee and hip joint replacement surgeries with discharge dates between 1 October 2015 and 2 October 2019. We tested whether people with HTN taking ACEis or ARBs undergoing these procedures had fewer complications after surgery when compared with those taking other antihypertensives. The outcomes included in the falsification analysis were obtained from a Centers for Medicare and Medicaid Services procedure-specific complication measure for hospitals and included the following complications: acute myocardial infarction or sepsis within 7 days; surgical site complication, pulmonary embolism or death within 30 days; and mechanical complication or periprosthetic wound infection/complication within 90 days. ${ }^{22}$ The original specification includes pneumonia as a complication, but because our primary study includes pneumonia, we excluded that outcome from the falsification analysis. We tested two outcomes: a composite measure of one or more complications other than death, and death in the same or following calendar month. As with the main analysis, we limited the cohort to people with at least 180 days of medical and pharmacy coverage before the index date and to people who met claims criteria for uncomplicated or complicated HTN. This analysis followed patients for up to 90 days; we therefore excluded patients with less than 90 days of continuous enrolment after surgery, unless the patient died during that time.

Stata/MP V.16.0 was used for all analyses (StataCorp College Station, Texas, USA, 2019). The lead author had complete access to the database and conducted all analyses. All authors had access to summary data and analysis output.

\section{RESULTS}

During the 2018-2019 influenza season, 236843 episodes of AVRI contributed by 202629 unique individuals were observed in the OLDW that met inclusion criteria for this study (cohort flow diagram provided in online supplemental figure 1). Similar proportions were associated with an ACEi (31.7\%) or an ARB (31.2\%) (table 1); somewhat more episodes were associated with other HTN medications $(37.1 \%)$. More than $80 \%$ of episodes were in people with MA insurance $(\mathrm{N}=192829 ; 81.4 \%)$. More than half of episodes were in female enrollees $(\mathrm{N}=139438 ; 58.9 \%)$ and white enrollees $(\mathrm{N}=139024 ; 58.7 \%)$. People aged 75 years and over contributed 95327 episodes (40.3\%).
All nine census divisions were represented, with the largest number of episodes in the South Atlantic division $(\mathrm{N}=93144 ; 39.3 \%)$.

There were differences in the demographic and clinical characteristics of patients across the medication groups (figures 1 and 2; underlying data in online supplemental table 2). The largest difference between the other HTN group and the group taking ACEis or ARBs was in the proportion with lymphoma: the ACEi or ARB group had $40 \%$ fewer people with lymphoma than the other HTN medications group (RR 0.60 (95\% CI 0.56 to 0.65$)$; adjusted $\mathrm{p}<0.001)$. People using ACEis or ARBs were more likely to have commercial insurance (RR 1.43 (95\% CI 1.30 to 1.46$)$ ), to have uncomplicated diabetes (RR 1.43 (95\% CI 1.40 to 1.46)), and to be of Hispanic ethnicity (RR 1.41 (95\% CI 1.38 to 1.45 )), and less likely to be aged 85 years or older (RR 0.65 (95\% CI 0.63 to $0.66)$ ), or to have metastatic cancer (RR 0.64 (95\% CI 0.61 to 0.68$)$ ), renal failure (RR 0.64 (95\% CI 0.63 to $0.65)$ ), liver failure (RR 0.65 (95\% CI 0.62 to 0.68$)$ ), congestive heart failure (RR 0.65 (95\% CI 0.64 to 0.66 )), complicated HTN (RR 0.72 (95\% CI 0.70 to 0.73 )), nonmetastatic solid tumour cancer (RR 0.73 (95\% CI 0.71 to $0.75)$ ), coronary artery disease (RR 0.79 (95\% CI 0.78 to $0.80)$ ), or chronic pulmonary disease (RR 0.80 (95\% CI 0.79 to 0.80$)$ ) than those using other HTN medications, all with adjusted $\mathrm{p}<0.001$.

Generally, the ACEi and ARB cohorts were more similar to each other than to the other HTN medications group, except for two characteristics: people taking ACEis were more likely to be under age 25 years (RR 2.49 (95\% CI 1.69 to 3.66)), and less likely to be Asian (RR 0.50 (95\% CI 0.47 to 0.54$)$ ) than those taking ARBs, both with adjusted $\mathrm{p}<0.001$.

Unadjusted outcome rates for AVRI are presented in table 2. Poor outcomes were somewhat more common among those taking no HTN medications and those taking HTN medications other than ACEis or ARBs compared with those taking ACEis or ARBs. Inpatient stays were common, with admission rates ranging from $11.6 \%$ among ARB users ( $\mathrm{N}=8565$ admissions) to $17.1 \%$ among those taking other HTN medications $(\mathrm{N}=15042)$. A relatively large proportion of the cohort died the same or the following calendar month: $4.2 \%$ of the included cohort $(\mathrm{N}=10015)$, including $2.9 \%(\mathrm{~N}=2128)$ of those using ARBs, 3.5\% ( $\mathrm{N}=2591)$ of those using ACEis, $6.0 \%$ $(\mathrm{N}=5296)$ of those using other HTN medications. In comparison, among people with AVRI meeting the claims definition for HTN but not using any HTN medications, $6.2 \%(\mathrm{~N}=2604)$ died the same or following calendar month.

With demographic and clinical differences across the HTN groups, unadjusted outcome rates are likely to be confounded. We conducted logistic regression analyses to estimate adjusted ORs for inpatient stay, ICU/CCU, acute respiratory distress, ARDS and death (figures 3 and 4 present ORs after logistic regression for the medication groups by outcome; complete regression result tables 
Table 1 Cohort characteristics; values are numbers (percentages) except where specified

Included cohort

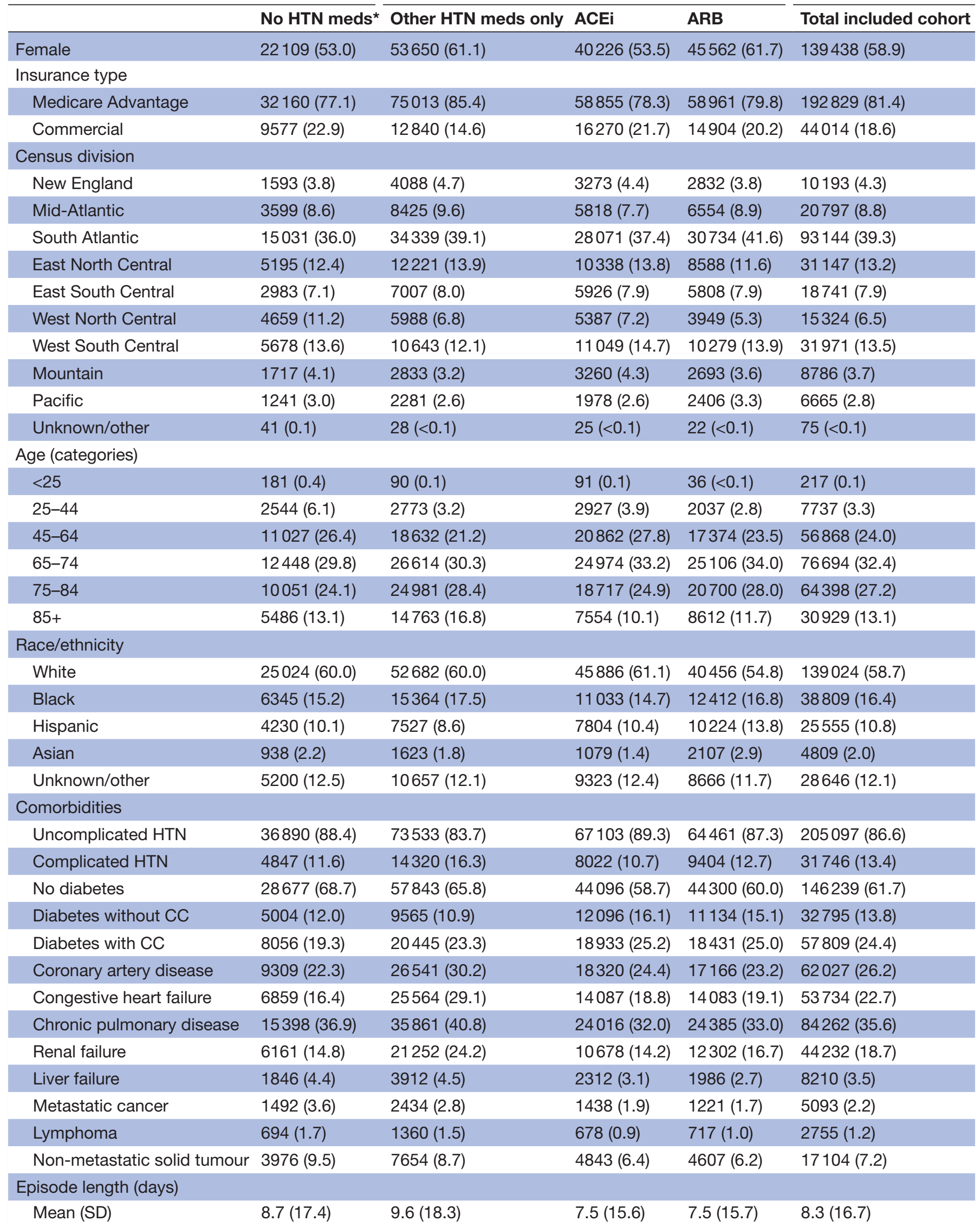


Table 1 Continued

\begin{tabular}{|c|c|c|c|c|c|}
\hline & \multirow[b]{2}{*}{ No HTN meds* } & \multicolumn{3}{|l|}{ Included cohort } & \multirow[b]{2}{*}{ Total included cohort } \\
\hline & & Other HTN meds only & ACEi & ARB & \\
\hline $\begin{array}{l}\text { Median (25th, 75th } \\
\text { percentiles) }\end{array}$ & $1(1,8)$ & $1(1,10)$ & $1(1,7)$ & $1(1,7)$ & $1(1,8)$ \\
\hline \multicolumn{6}{|c|}{ Number of days of healthcare with HTN diagnosis, 180 days before index date } \\
\hline Mean (SD) & $5.0(7.2)$ & $6.0(7.2)$ & $5.0(5.9)$ & $5.0(5.6)$ & $5.4(6.4)$ \\
\hline $\begin{array}{l}\text { Median (25th, 75th } \\
\text { percentiles) }\end{array}$ & $3(2,5)$ & $4(2,7)$ & $3(2,5)$ & $3(2,6)$ & $3(2,6)$ \\
\hline N (episodes) & 41737 & 87853 & 75125 & 73865 & 236843 \\
\hline $\mathrm{N}$ (unique people) & 38011 & 75815 & 66283 & 63941 & 202629 \\
\hline
\end{tabular}

${ }^{*}$ This group is not included in analyses; characteristics are presented for context.

ACEi, ACE inhibitor; ARB, angiotensin receptor blocker; CC, complicating condition; HTN, hypertension.

are available in online supplemental table 3). Odds of mortality were lower in both the ACEi (OR 0.78 (95\% CI 0.74 to 0.83 ) ) and ARB (OR 0.64 (95\% CI 0.61 to 0.68$)$ ) cohorts compared with other HTN medications. On all other outcomes, people taking ARBs had a $>10 \%$ reduction in odds of inpatient stays (OR 0.82 (95\% CI 0.79 to 0.84$)$ ), ICU/CCU (OR 0.82 (95\% CI 0.79 to 0.86 )), acute respiratory distress (OR 0.78 (95\% CI 0.70 to 0.87 )) and ARDS (OR 0.70 (95\% CI 0.58 to 0.85)) compared with other HTN medications. For outcomes other than mortality, people taking ACEis had similar odds as people taking other HTN medications (ORs all between 0.93 and $0.96)$.

\section{Falsification analyses result}

We assessed complications and deaths in a cohort of people receiving hip or knee replacement surgery, comparing outcomes across the HTN medication groups as a falsification analysis for the main cohort of people with AVRI. The study sample included 103353 surgeries (93714 unique people). A cohort flow diagram (Figure B) and table of cohort demographics (Table D) are provided

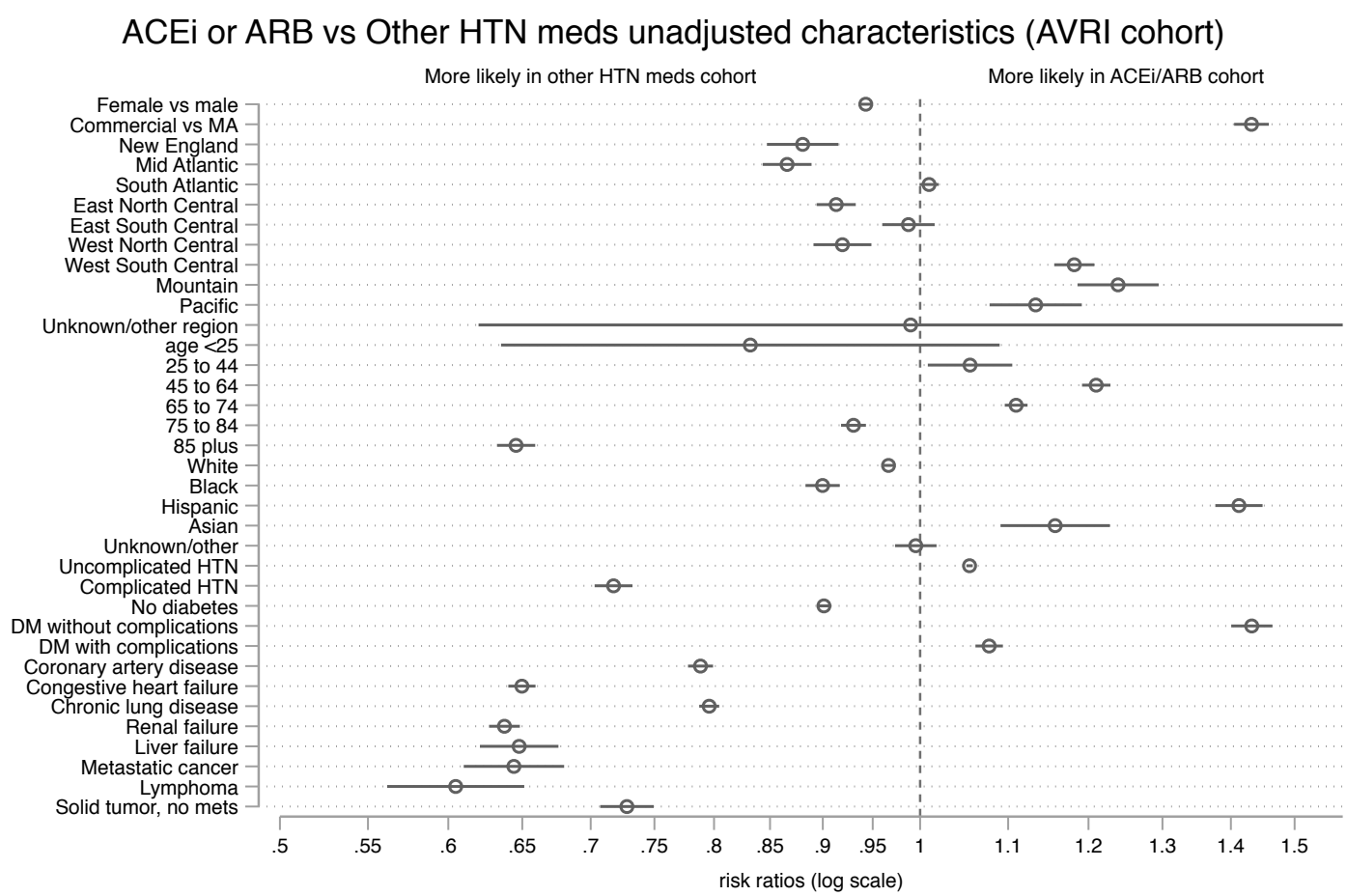

Figure 1 Comparisons of cohort characteristics. Rate ratios comparing baseline characteristics of people taking ACEis or ARBs with people taking other HTN medications; the comparison for age 25-44 years is not statistically significant after multiple comparison adjustment (unadjusted $\mathrm{p}=0.02$; adjusted $\mathrm{p}=0.24$ ). ACEi, ACE inhibitor; $\mathrm{ARB}$, angiotensin receptor blocker; DM, diabetes mellitus; HTN, hypertension; MA, Medicare Advantage. 


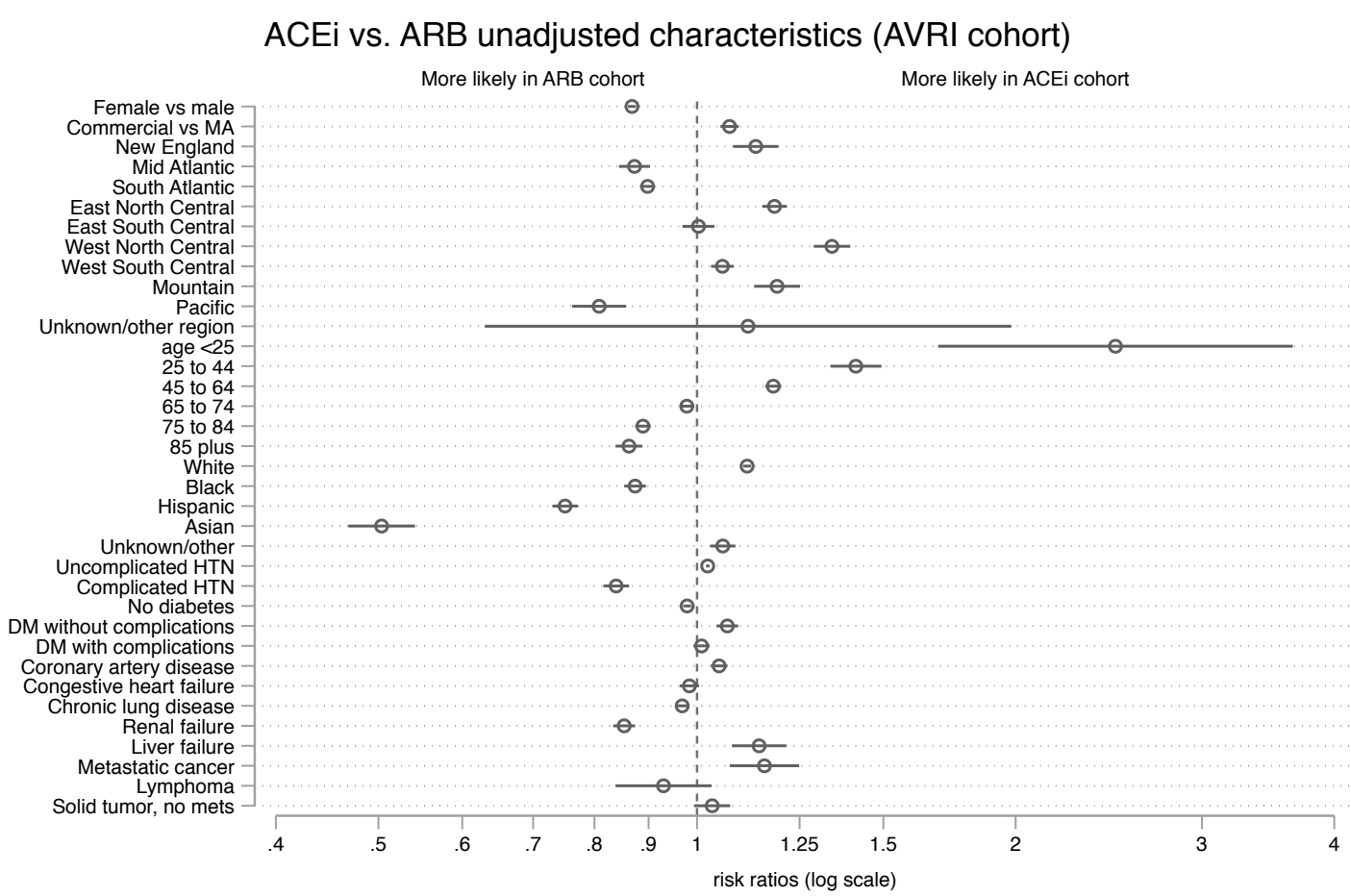

Figure 2 Comparisons of cohort characteristics. Rate ratios comparing baseline characteristics of people taking ACEis with people taking ARBs. ACEi, ACE inhibitor; ARB, angiotensin receptor blocker; DM, diabetes mellitus; HTN, hypertension; MA, Medicare Advantage.

in the supplement. Comparisons of medication cohort demographic and clinical characteristics similar to those in figures 1 and 2 are provided in online supplemental figure 2 and online supplemental table 5. Demographic comparisons between the groups were broadly similar to those in the AVRI cohort, for example, people in the ARB group were much more likely to be Asian than people in the ACEi group. Complications were rare in the falsification analysis cohort (table 3).
Figure 4 presents adjusted ORs after logistic regression for two outcomes: the composite outcome measuring whether a patient had one or more of a list of complications, and the mortality outcome measuring whether a person died in the same or the following calendar month of their surgery. Complete regression results are available in online supplemental table 4. Both the ACEi and ARB cohorts had statistically significantly lower odds of the composite outcome of non-death complications

Table 2 Unadjusted outcome rates for acute viral respiratory illness (AVRI) cohort by HTN medication group; values are numbers of episodes (percentages)

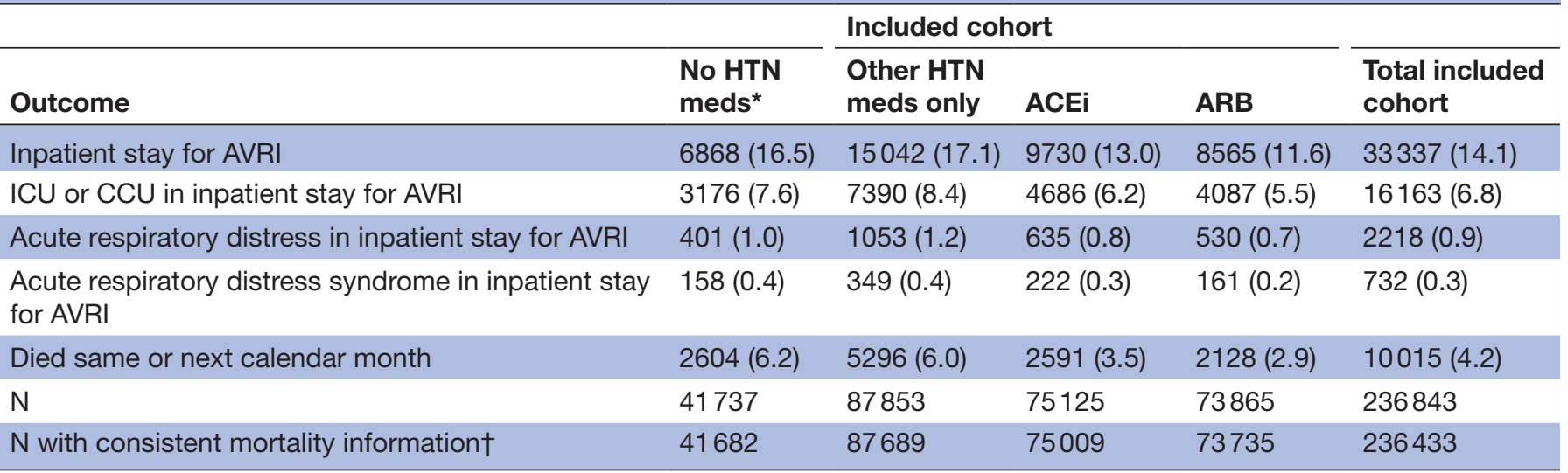

*This group is not included in analyses; characteristics are presented for context.

†Denominator for death outcome excludes 410 people with inconsistent mortality information (date of death more than 1 calendar month before AVRI episode ends).

ACEi, ACE inhibitor; ARB, angiotensin receptor blocker; CCU, coronary care unit; HTN, hypertension; ICU, intensive care unit. 
AVRI cohort: adjusted odds ratios

More likely in other HTN meds cohort

More likely in ACEi/ARB cohort

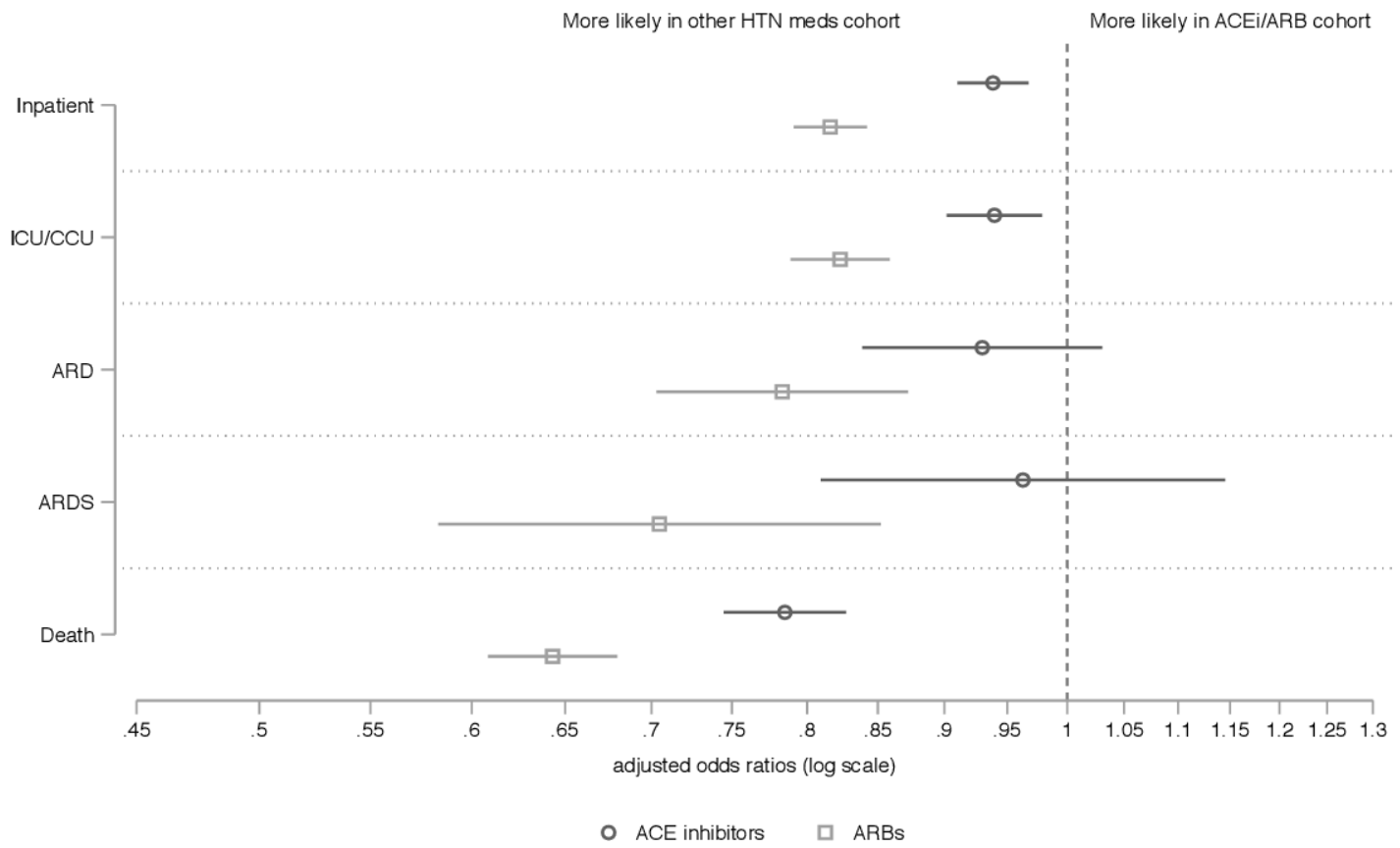

Figure 3 Adjusted ORs for five outcomes in AVRI cohort; ORs compare odds of outcome in ACEi cohort with those in the other HTN medication cohort. Each logistic regression included the following covariates: gender, age (categorical), insurance type (Medicare Advantage vs commercial), census division, race/ethnicity, diabetes (none, without complications, with complications), HTN (without complications, with complications), CAD, CHF, chronic pulmonary disease, renal failure, liver failure, metastatic cancer, lymphoma and non-metastatic solid tumour. ACEi, ACE inhibitor; ARB, angiotensin receptor blocker; $A R D$, acute respiratory distress; ARDS, acute respiratory distress syndrome; AVRI, acute viral respiratory illness; CAD, coronary artery disease; CCU, coronary care unit; CHF, congestive heart failure; HTN, hypertension; ICU, intensive care unit.

compared with the other HTN medications cohort (ACEi: OR 0.89 (95\% CI 0.80 to 0.98); ARB: OR 0.82 (95\% CI 0.74 to 0.91$)$ ). The ARB cohort but not the ACEi cohort had statistically significantly lower odds of death in the same or following calendar month as the surgery (ARB: OR 0.64 (95\% CI 0.47 to 0.87); ACEi: OR 0.79 (95\% CI 0.60 to 1.05$)$ ) compared with the other HTN medications group.

\section{DISCUSSION}

In this observational study, we found that patients with an AVRI who were taking ACEis or ARBs for management of their HTN had a lower risk of death in the same or following calendar month as their episode of illness compared with patients on other antihypertensive medications like beta-blockers, diuretics or calcium channel blockers. People taking ARBs also had a statistically significant reduced risk of hospitalisation, ICU or CCU stays, acute respiratory distress and ARDS during that episode of illness. The ACEi group had similar outcomes to the other HTN medications group on all outcomes other than death, with OR point estimates close to 1 .

In the falsification cohort, point estimates for ORs for both mortality and the composite complication outcome were lower in the ARB than the ACEi cohort, but the outcomes were rare and ORs were not statistically distinguishable from each other in either outcome.
Broadly speaking, the falsification test fails: the risk of poor outcomes after knee or hip replacement was lower in the ACEi and ARB cohorts than the cohort taking other HTN medications. Furthermore, in the mortality outcome measured in both the AVRI and falsification cohorts, the OR point estimates were nearly identical across the two analyses: the ACEi OR was 0.78 in the AVRI cohort and 0.79 in the falsification cohort; the ARB OR was 0.64 in both cohorts.

The case of post-surgical complications after hip or knee replacement was intended to provide an example of a medical condition unlikely to be affected by the hypothesised mechanism for ACEis and ARBs in AVRI. The failure of the falsification test may suggest that there are unmeasured differences in the cohorts that favour the ACEi and ARB groups or that there may also be a protective effect of these two drug classes in hip and knee surgery relative to other HTN medications. It is possible that the RAAS is stimulated by elective surgery and perhaps the same mechanistic protective effects could apply to those conditions, or any protective effect may not be related to the RAAS but other properties of these medicines like their antifibrotic activity, wound healing and local inflammation properties. This study cannot distinguish between the possibility of a true protective effect in the surgery cohort versus uncontrolled confounding to explain the results of the falsification analysis. 
Falsification cohort: adjusted odds ratios

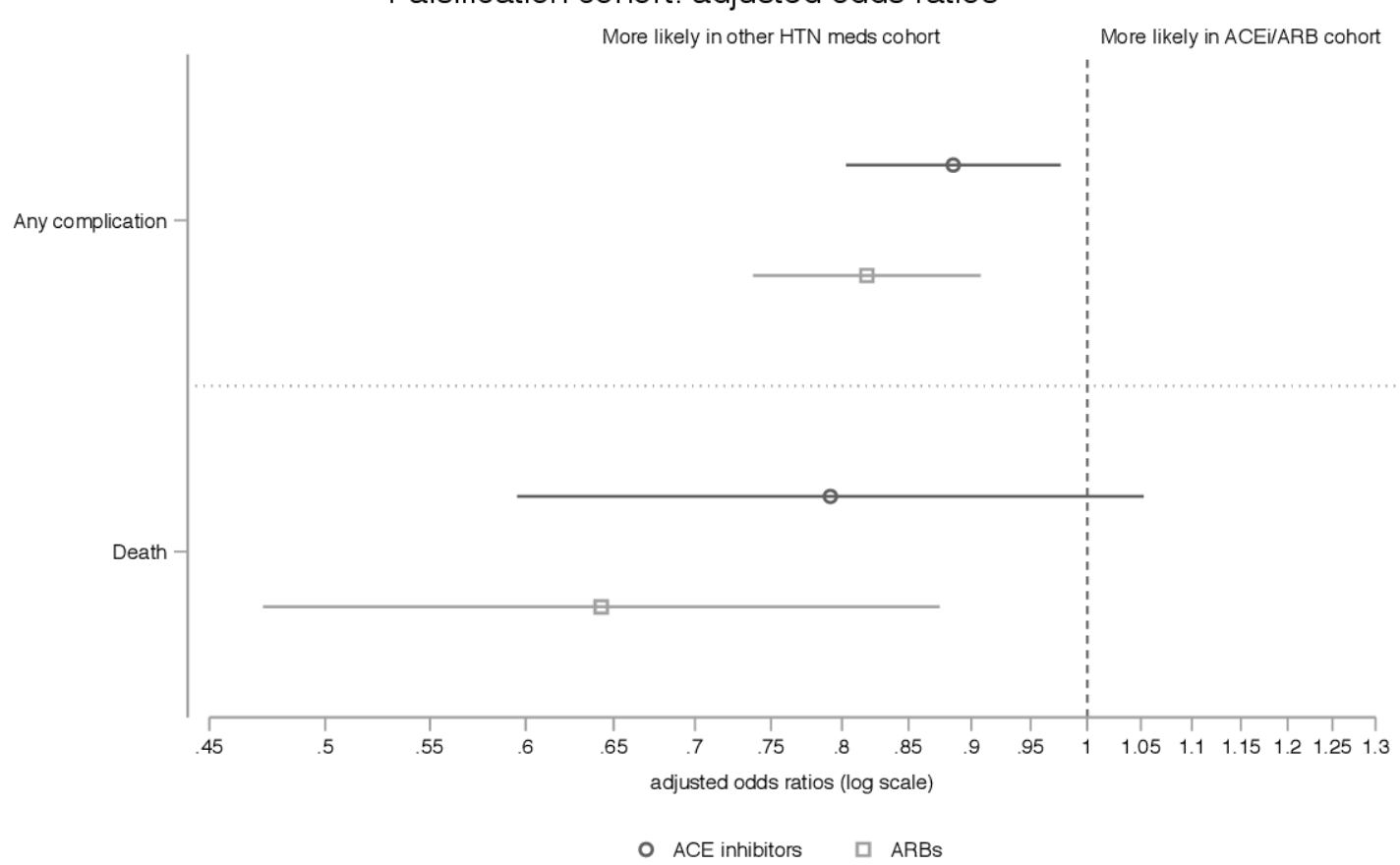

Figure 4 Adjusted ORs for two outcomes in falsification (hip/knee replacements) cohort; ORs compare odds of outcome in ACEi cohort with those in the other HTN medication cohort. Each logistic regression included the following covariates: gender, age (categorical), insurance type (Medicare Advantage vs commercial), census division, race/ethnicity, diabetes (none, without complications, with complications), HTN (without complications, with complications), CAD, CHF, chronic pulmonary disease, renal failure, liver failure, metastatic cancer, lymphoma and non-metastatic solid tumour. ACEi, ACE inhibitor; ARB, angiotensin receptor blocker; CAD, coronary artery disease; CHF, congestive heart failure; HTN, hypertension.

In both the AVRI and the falsification cohorts, we found that patients taking ACEis or ARBs were somewhat younger, more likely to have commercial insurance and less likely to have some serious comorbidities including metastatic cancer, non-metastatic solid tumours, liver failure, renal failure, coronary artery disease or congestive heart failure than those using other HTN medications. These underlying health differences and others we were not able to measure may in part explain the beneficial effects associated with ACEis and ARBs in this and other observational studies of outcomes in respiratory illnesses, including COVID-19. For example, in a study of VA inpatients with pneumonia or influenza, Mortensen $e t a l^{23}$ found that statins, ACEis and ARBs were associated with decreased mortality and length of stay compared with a propensity score-matched cohort not taking those medications. Shah et $a l^{24}$ assessed risk of hospitalisation for pneumonia in the first 90 days of various HTN medications. Compared with calcium channel blockers, both ACEis and ARBs were associated with reduced risk of the outcome.

Table 3 Unadjusted outcome rates for falsification (elective knee or hip replacement) cohort by HTN medication group; values are numbers of episodes (percentages)

\begin{tabular}{|c|c|c|c|c|c|}
\hline & & Included cohort & & & \\
\hline Outcome & No HTN meds* & Other HTN meds only & ACEi & ARB & Total included cohor \\
\hline Any complication $†$ & $407(2.1)$ & $851(2.7)$ & $892(2.3)$ & $696(2.1)$ & $2439(2.4)$ \\
\hline $\mathrm{N}$ & 19087 & 31659 & 39026 & 32668 & 103353 \\
\hline $\begin{array}{l}\mathrm{N} \text { with consistent mortality } \\
\text { information } \ddagger\end{array}$ & 19059 & 31603 & 38964 & 32616 & 103183 \\
\hline
\end{tabular}

${ }^{*}$ This group is not included in analyses; characteristics are presented for context.

†Acute myocardial infarction or sepsis within 7 days of surgery; surgical site complication or pulmonary embolism within 30 days; and/or mechanical complication or periprosthetic wound infection/complication within 90 days.

$\ddagger$ Denominator for death outcome excludes 170 people with inconsistent mortality information (date of death more than 1 calendar month before surgery).

ACEi, ACE inhibitor; ARB, angiotensin receptor blocker; HTN, hypertension. 
Beta-blockers and thiazide diuretics were not associated with a reduced risk of pneumonia hospitalisation. A recent study including 1128 patients with HTN and COVID-19, including 188 taking ACEi/ARB, found that among hospitalised patients, inpatient use of ACEi/ARB was associated with lower risk of all-cause mortality compared with ACEi/ARB non-users. ${ }^{25}$ An observational study from China including 476 patients with COVID-19 found more patients taking ACEi/ARB in the moderate disease severity group compared with severe and critical groups; however, the total number of patients on ACEi or ARB was very small ( $n=33$ of the 113 who had HTN).${ }^{26}$ Another observational study with 417 patients with COVID-19 including 17 on ACEi/ ARBs concluded using ACEis or ARBs potentially contributed to the improvement of clinical outcomes of patients with COVID-19 with HTN. ${ }^{27}$ Serum angiotensin II levels were significantly higher in COVID-19infected patients and had an association with viral load and lung damage in one study. ${ }^{28}$ This can increase pulmonary vasoconstriction and lead to ventilation/ perfusion mismatch, increased vascular permeability, inflammation and oxidation, and ultimately the development of ARDS. ${ }^{129}$ However, it is important to note that none of these studies used methods to assess the causal implications of differences in outcomes. The Mortensen study used propensity score matching to create a cohort balanced on observed characteristics, but propensity score methods, like regression methods, are not able to control for unmeasured confounders. ${ }^{23}$ Our results suggest that unmeasured differences between medication cohorts may contribute to identified associations between ACEis and ARBs and improved outcomes with acute illness or surgery.

\section{Limitations}

This study is limited to prescription fills submitted for insurance payment and to a population of commercial and MA beneficiaries. We do not include the uninsured, or people with Medicaid or fee-for-service Medicare. This study looks at the long-term effect of these medications in patients taking ACEis/ARBs prior to getting sick; we are not able to use claims data to test ACEi/ARB as an interventional treatment for people who have not received them before. Prospective trials are necessary to establish whether starting ACEis/ARBs has therapeutic benefit for AVRI.

We have controlled for measured confounders but cannot eliminate all potential confounders, and regression cannot completely control for measured confounders. In particular, we do not assess blood pressure control and are unable to determine whether any of the medication cohorts have better control, which could in turn systematically affect mortality. Finally, our mortality data are limited in detail (month of death only) and rely in part on data from the Social Security Death Master File, which is an incomplete record of all US deaths. ${ }^{30}$ However, these data have been supplemented with mortality information from hospital discharge information and insurance enrolment records.

\section{CONCLUSION}

Chronic outpatient use of ARBs was associated with better outcomes with AVRI compared with other medications for HTN. Chronic use of ACEis was associated with reduced risk of death with AVRI, but with minimal or no reduction in risk of other complications. A falsification analysis conducted to provide context on the possible causal implications of these findings failed-ACEis and ARBs were associated with reductions in the odds of complications and death compared with the other HTN medications group. Future work using observational data to assess causal relationships between these drugs and outcomes in AVRI must consider alternative approaches to assess the importance of confounding in these relationships.

\section{Author affiliations}

${ }^{1}$ Division of Health Care Delivery Research, Mayo Clinic, Rochester, Minnesota, USA

${ }^{2}$ Emergency Medicine, Mayo Clinic, Rochester, Minnesota, USA

${ }^{3}$ Department of Medicine, Division of Infectious Diseases, Mayo Clinic, Rochester,

Minnesota, USA

${ }^{4}$ Pulmonary Critical Care Medicine, David Grant Medical Center, Travis AFB,

California, USA

${ }^{5}$ Division of Pulmonary and Critical Care Medicine, Mayo Clinic, Rochester, Minnesota, USA

${ }^{6}$ Robert D and Patricia E Kern Center for the Sciences of the Health Care Delivery, Mayo Clinic, Rochester, Minnesota, USA

Twitter Molly Moore Jeffery @mollyjeffery and Fernanda Bellolio @mfbellolio Contributors MMJ, FB and NWC conceived and designed this work. MMJ cleaned and analysed the data. MMJ, NWC, TMD, AL, NDS and FB interpreted the data; while MMJ, NWC, TMD, AL, NDS and FB were responsible for drafting the work and revising it critically for important intellectual content. MMJ, NWC, TMD, AL, NDS and FB gave final approval of the version to be published and agree to be accountable for all aspects of the work in ensuring that questions related to the accuracy or integrity of any part of the work are appropriately investigated and resolved. MMJ is the guarantor.

Funding The authors have not declared a specific grant for this research from any funding agency in the public, commercial or not-for-profit sectors.

Competing interests All authors have completed the ICMJE uniform disclosure form at www.icmje.org/coi_disclosure.pdf (available on request from the corresponding author) and declare: no support from any organisation for the submitted work; no financial relationships with any organisations that might have an interest in the submitted work in the previous 3 years; no other relationships or activities that could appear to have influenced the submitted work.

Patient consent for publication Not required.

Ethics approval Because this study uses pre-existing de-identified data, it was determined to be exempt from review by the Mayo Clinic Institutional Review Board.

Provenance and peer review Not commissioned; externally peer reviewed.

Data availability statement Data may be obtained from a third party and are not publicly available. OptumLabs data are available for research through a virtual data warehouse. The authors are not able to distribute the data.

Supplemental material This content has been supplied by the author(s). It has not been vetted by BMJ Publishing Group Limited (BMJ) and may not have been peer-reviewed. Any opinions or recommendations discussed are solely those of the author(s) and are not endorsed by BMJ. BMJ disclaims all liability and responsibility arising from any reliance placed on the content. Where the content includes any translated material, BMJ does not warrant the accuracy and reliability of the translations (including but not limited to local regulations, clinical guidelines, 
terminology, drug names and drug dosages), and is not responsible for any error and/or omissions arising from translation and adaptation or otherwise.

Open access This is an open access article distributed in accordance with the Creative Commons Attribution Non Commercial (CC BY-NC 4.0) license, which permits others to distribute, remix, adapt, build upon this work non-commercially, and license their derivative works on different terms, provided the original work is properly cited, appropriate credit is given, any changes made indicated, and the use is non-commercial. See: http://creativecommons.org/licenses/by-nc/4.0/.

\section{ORCID iD}

Molly Moore Jeffery http://orcid.org/0000-0003-3854-6810

\section{REFERENCES}

1 Sanchis-Gomar F, Lavie CJ, Perez-Quilis C, et al. AngiotensinConverting enzyme 2 and antihypertensives (angiotensin receptor blockers and angiotensin-converting enzyme inhibitors) in coronavirus disease 2019. Mayo Clin Proc 2020;95:1222-30.

2 Kickbusch I, Leung G. Response to the emerging novel coronavirus outbreak. BMJ 2020;368:m406.

3 American Heart Association, Heart Failure Society of America, American College of Cardiology. Patients taking ACE-i and Arbs who contract COVID-19 should continue treatment, unless otherwise advised by their physician 2020, 2020. Available: https://newsroom. heart.org/news/patients-taking-ace-i-and-arbs-who-contract-covid19-should-continue-treatment-unless-otherwise-advised-by-theirphysician/

4 European Society of Hypertension. Statement of the European Society of hypertension (ESH) on hypertension, renin-angiotensin system (ras) blockers and COVID-19 2020, 2020. Available: https:// www.eshonline.org/esh-content/uploads/2020/06/Statement-ESHon-Hypertension-RAS-Blockers-and-COVID-19-Update-April-152020.pdf

5 European Society of Cardiology Council on Hypertension. Position statement of the ESC Council on hypertension on ACE-inhibitors and angiotensin receptor blockers 2020, 2020. Available: https:// www.escardio.org/Councils/Council-on-Hypertension-(CHT)/News/ position-statement-of-the-esc-council-on-hypertension-on-aceinhibitors-and-ang

6 International Society of Hypertension. A statement from the International Society of hypertension on COVID-19, 2020. Available: https://ish-world.com/news/a/A-statement-from-the-InternationalSociety-of-Hypertension-on-COVID-19/

7 Khan N. Hypertension Canada's statement on: hypertension, ACEinhibitors and angiotensin receptor blockers and COVID-19, 2020. Available: https://hypertension.ca/wp-content/uploads/2020/03/ 2020-30-15-Hypertension-Canada-Statement-on-COVID-19-ACEiARB.pdf

8 Mackey K, King VJ, Gurley S, et al. Risks and impact of angiotensinconverting enzyme inhibitors or angiotensin-receptor blockers on SARS-CoV-2 infection in adults: a living systematic review. Ann Intern Med 2020;173:195-203.

9 Vaduganathan M, Vardeny O, Michel T, et al. Renin-AngiotensinAldosterone system inhibitors in patients with Covid-19. N Engl $J$ Med 2020;382:1653-9.

10 Tignanelli CJ, Ingraham NE, Sparks MA, et al. Antihypertensive drugs and risk of COVID-19? Lancet Respir Med 2020;8:e30-1.
11 Kuba K, Imai Y, Rao S, et al. A crucial role of angiotensin converting enzyme 2 (ACE2) in SARS coronavirus-induced lung injury. Nat Med 2005;11:875-9.

12 Yang P, Gu H, Zhao Z, et al. Angiotensin-Converting enzyme 2 (ACE2) mediates influenza H7N9 virus-induced acute lung injury. Sci Rep 2014;4:7027.

13 Benchimol El, Smeeth L, Guttmann A, et al. The reporting of studies conducted using observational Routinely-collected health data (record) statement. PLoS Med 2015;12:e1001885.

$14 \mathrm{Xu} \mathrm{X,} \mathrm{Blanton} \mathrm{L,} \mathrm{Elal} \mathrm{AIA,} \mathrm{et} \mathrm{al.} \mathrm{Update:} \mathrm{influenza} \mathrm{activity} \mathrm{in} \mathrm{the}$ United States during the 2018-19 season and composition of the 2019-20 influenza vaccine. MMWR Morb Mortal Wkly Rep 2019;68:544-51.

15 Wallace PJ, Shah ND, Dennen T, et al. Optum Labs: building a novel node in the learning health care system. Health Aff 2014;33:1187-94.

16 Quan $\mathrm{H}$, Sundararajan V, Halfon P, et al. Coding algorithms for defining comorbidities in ICD-9-CM and ICD-10 administrative data. Med Care 2005;43:1130-9.

17 Noseworthy PA, Gersh BJ, Kent DM, et al. Atrial fibrillation ablation in practice: assessing CABANA generalizability. Eur Heart $J$ 2019;40:1257-64.

18 Papadakis MA, McPhee SJ, Rabow MW. Current medical diagnosis \& treatment. 55th ed. New York: McGraw-Hill Education, 2016.

19 Holm S. A simple sequentially rejective multiple test procedure. Scandinavian Journal of Statistics 1979;6:65-70.

20 Aickin M, Gensler $\mathrm{H}$. Adjusting for multiple testing when reporting research results: the Bonferroni vs Holm methods. Am J Public Health 1996;86:726-8.

21 Prasad V, Jena AB. Prespecified falsification end points: can they validate true observational associations? JAMA 2013;309:241-2.

22 Yale New Haven Health Services Corporation - Center for Outcomes Research \& Evaluation. 2019 Procedure-Specific complication measure updates and specifications ReportElective primary total hip arthroplasty (THA) and/or total knee arthroplasty (TKA) - version 8.0, 2019. Available: https://www.qualitynet.org/inpatient/measures/ complication/resources\#tab3

23 Mortensen EM, Nakashima B, Cornell J, et al. Population-based study of statins, angiotensin II receptor blockers, and angiotensinconverting enzyme inhibitors on pneumonia-related outcomes. Clin Infect Dis 2012;55:1466-73.

24 Shah S, McArthur E, Farag A, et al. Risk of hospitalization for community acquired pneumonia with renin-angiotensin blockade in elderly patients: a population-based study. PLoS One 2014;9:e110165.

25 Zhang P, Zhu L, Cai J, et al. Association of inpatient use of angiotensin-converting enzyme inhibitors and angiotensin II receptor blockers with mortality among patients with hypertension hospitalized with COVID-19. Circ Res 2020;126:1671-81.

26 Feng Y, Ling Y, Bai T, et al. COVID-19 with different severities: a multicenter study of clinical features. Am J Respir Crit Care Med 2020;201:1380-8.

27 Meng J, Xiao G, Zhang J, et al. Renin-Angiotensin system inhibitors improve the clinical outcomes of COVID-19 patients with hypertension. Emerg Microbes Infect 2020;9:757-60.

28 Liu Y, Yang Y, Zhang C, et al. Clinical and biochemical indexes from 2019-nCoV infected patients linked to viral loads and lung injury. Sci China Life Sci 2020;63:364-74.

29 Zhang H, Baker A. Recombinant human ACE2: acing out angiotensin II in ARDS therapy. Crit Care 2017;21:305.

30 da Graca B, Filardo G, Nicewander D. Consequences for healthcare quality and research of the exclusion of records from the death master file. Circ Cardiovasc Qual Outcomes 2013;6:124-8. 